\title{
Una visión elsteriana de la modificación del comportamiento en México
}

\author{
José Carlos Vázquez Parra* \\ ITESM, CAMPUS GUADALAJARA
}

En los últimos ańos, parece ser que el aumento de la violencia en el país está llevando a que la población genere nuevas opciones de actuación. Sin embargo, al haber nacido como resultado del miedo, muchos de estos nuevos comportamientos tienen una fundamentación distorsionada, lo que nos lleva a la necesidad de adentrarnos en postulados, que como la propuesta elsteriana, busquen resarcir tales errores, pretendiendo que nuestras acciones sean cada vez más conscientes y racionales.

(Racionalidad, creencias, acción, conducta, deseo)

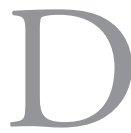

esde el año 2006, cuando el gobierno mexicano declaró el inicio de una guerra contra el crimen organizado, han fallecido alrededor de 60 mil personas a causa de ejecuciones directas, confrontaciones entre grupos rivales o bien con la autoridad (Mendoza 2011). Sin embargo, cabe señalar que aunque este enfrentamiento comenzó como una lucha del Estado mexicano por recuperar su soberanía ante el flagelo del crimen, parece ser que se ha abierto una caja de Pandora, saliendo de ella todas las calamidades que un Estado moderno puede temer.

De esta manera, los altos niveles de violencia que se vivían en ciertas zonas focalizadas del país se extendieron hacia todas las direcciones, afectando tanto zonas rurales como las urbes más importantes del país. Ciudades como Juárez, Torreón, Monterrey, Morelia,

\footnotetext{
*Jvazquez24hrs@gmail.com
} 
Acapulco, Tepic, entre otras, han sido fuertemente golpeadas por el crimen organizado, cambiando su popularidad de centros productivos o turísticos por una fama de peligro, violencia y muerte. Esto ha llevado a que algunos estados del país como Chihuahua, Sinaloa y Guerrero sean considerados dentro de las regiones más peligrosas del mundo, afectando la percepción global e interna de México (BBC 2011).

La Procuraduría General de la Republica (PGR) no teme en señalar que 9 de cada 10 de las víctimas de esta guerra son miembros del crimen organizado, lo que sugiere que $10 \%$ restante son personas que no tienen relación con estos enfrentamientos, es decir, civiles que tuvieron el infortunio de estar en el lugar equivocado en el momento equivocado. Esta situación ha hecho que la seguridad de la población mexicana se convierta en un tema de discusión en las diferentes mesas de debate, aunque mientras se discute acerca de la culpabilidad o justificación del Estado y las fuerzas armadas, cada día se suman más muertes, aumentando de esta manera los llamados daños colaterales de la llamada guerra contra el narcotráfico (Ballinas 2010).

Lo anterior es lo que ha ocasionado que el caminar por ciertas ciudades de México ya no sea tan seguro como era hace pocos años, pues si a los cotidianos peligros que conlleva la vida en grandes urbes poblacionales le sumamos el riesgo de quedar envuelto en un enfrentamiento armado, que se vuelve cada día más común, la posibilidad de no volver a casa comienza a vislumbrarse como un riesgo real, lo cual afecta completamente a cualquier persona. Esta situación ha llevado a que los ciudadanos tomen medidas para protegerse, las cuales incluyen desde el resguardo en sus hogares, hasta la modificación perceptible de sus hábitos comportamentales, de ocio y recreo.

Cada vez son menos las reuniones entre amigos, los eventos familiares han dejado de ser habituales y algunas de las zonas que albergaban la vida nocturna y de ocio de ciertas ciudades se han convertido en lugares sombríos, abandonados y sin vida. La población teme estar en las calles, pues, la tranquilidad parece romperse con mayor frecuencia por disparos lejanos o el ruido de las torretas. Pero, ¿̇hasta dónde se ve afectado el comportamiento y las acciones 
de la población?, ¿qué tan óptimos son tales comportamientos defensivos o las creencias que están respaldando dichas acciones?

Cuando un individuo vive una situación de amenaza o riesgo, todas sus acciones se apegarán a la urgencia de dicho escenario, sin embargo, cuando las circunstancias dejan de ser un momento y se vuelven en una realidad casi cotidiana, las opciones de actuación pueden verse gravemente modificadas. Esta situación da paso a elecciones, que bajo otros panoramas podrían considerarse como resultado de una psicosis colectiva, pero que en dicho momento y lugar, se convierten en la acción aparentemente más racional.

Según la Teoría Elsteriana de la Racionalidad, toda acción para ser considerada racional debe sustentarse en dos elementos básicos, un deseo racionalmente autónomo y creencias racionales que se basen en evidencia óptima. Sin embargo, ¿qué sucede cuando una situación como la que se vive en México llega a internalizarse en las creencias de sus ciudadanos?, ¿qué acciones pueden esperarse de una población que por medio de un proceso deliberativo hace parte de sus creencias el vivir en una realidad violenta, peligrosa y amenazante?, ¿qué tanto pueden verse afectadas sus preferencias, su elección e incluso sus acciones en general?, ¿qué tan racionales siguen siendo sus comportamientos?

\section{LA REALIDAD}

El 11 de diciembre del 2006, el gobierno de México anunció que emprendería una batalla contra el crimen organizado, es decir, que enfrentaría a los diferentes grupos delictivos que controlaban la mayoría de las actividades ilegales del país, principalmente, el narcotráfico, el secuestro, la extorsión y el tráfico de armas. Para esta cruenta y complicada batalla, el gobierno federal requirió sacar a las fuerzas armadas de los cuarteles y lo que en un inicio parecería un plan de seguridad, poco a poco se fue asemejando a una guerra interna en la que el ejército y la marina desempeñan un papel fundamental.

Conforme avanzaron los meses, las calles de diferentes ciudades del país se fueron convirtiendo en el campo de esta batalla, la cual, aunque daba resultados en cuanto al número de detenciones, tam- 
bién arrojaba un saldo rojo muy alarmante. Por otro lado, lo que en un inicio era una lucha entre el Estado y los grupos del crimen organizado, rápidamente fue dando lugar a disputas dentro de las mismas bandas, generando nuevas situaciones de riesgo para la población, que en esta guerra quedaba en medio del fuego cruzado.

El número de civiles, es decir, aquellos que no tienen relación con actividades delictivas, que han sido asesinados durante los diferentes enfrentamientos ha llegado a miles, desencadenando fuertes criticas de diferentes organizaciones no gubernamentales, tanto nacionales como internacionales, a esta lucha que parece no mostrar un pronto desenlace. Los medios de comunicación, las estadísticas y la opinión pública se han volcado en señalar el incremento aterrador de la violencia en México y aunque el Poder Ejecutivo Nacional busque frenar esta campaña de desprestigio, la realidad se vuelve en su peor enemigo, pues no pasa un día sin haber nuevas noticias de conflicto, violencia y muerte.

Los ataques a la población civil parece que cada vez se hacen más cotidianos y aunque, posteriores a ellos, los diferentes grupos del crimen organizado se deslinden de responsabilidades y señalen que el matar civiles no es su intención, la situación que se ha generado es de una alarma y un miedo generalizado entre la ciudadanía. Sucesos como el ataque calificado como narcoterrorista (El Universal 2008) con granadas en la plaza central de Morelia, Michoacán, durante las festividades del grito de independencia, fueron ocasionando que la población de ciertas ciudades fuera perdiendo su tranquilidad en espacios públicos.

A este suceso, se le sumó la posterior muerte de dos estudiantes del Instituto Tecnológico y de Estudios Superiores de Monterrey, Campus Monterrey, en un fuego cruzado entre militares y miembros del crimen organizado en el 2010, así como la matanza ocurrida en el 2011 en la que individuos de un grupo delictivo incendió un casino de la ciudad de Monterrey, Nuevo León, asesinando a 52 personas, entre ellas una mujer embarazada.

Estas lamentables situaciones se han vuelto una realidad entre la población de México, la cual se ha dado cuenta de que ya no es suficiente mantenerse al margen de los grupos delictivos o evitar ciertas 
zonas de peligro, pues aun en las actividades más cotidianas se puede estar en riesgo. Éste es el México del 2012, en el cual, sin importar la hora, el género, la edad, el lugar o el entorno, se puede quedar en medio de esta batalla en la que la población termina siendo la más afectada.

\section{ConseCUENCIAS EN EL COMPORTAMIENTO DE LA POBLACIÓN}

Ataques de diferentes índoles, secuestros, robo de automóviles, extorsión, enfrentamientos entre carteles, narcobloqueos, entre otras situaciones de riesgo, parece que están modificando la vida y las acciones de los ciudadanos de algunas de las ciudades más importantes del país, dando lugar a nuevas costumbres, creencias y preferencias de actuación, que antes no se hubieran imaginado, pero que hoy son claramente palpables.

El resguardo de la información personal, el uso de medios informáticos para prevenir situaciones de riesgo, el evitar espacios públicos donde haya gran afluencia de personas, son algunas de las previsiones que un ciudadano comienza a hacer parte de sus creencias, dirigiendo sus actuaciones hacia actividades que le permitan volver a su casa sano y salvo, así como proteger a los suyos ante una realidad desafiante.

En ciudades como Monterrey, este cambio de comportamiento se ha vuelto muy notorio, reduciendo de manera muy considerable la vida nocturna de una de las ciudades más importantes del país. Según algunos medios, entre 30 y $40 \%$ de los negocios del centro histórico de esta ciudad han cerrado por las bajas ventas de los últimos meses, así como por el flagelo constante de los diferentes grupos delictivos (Hernández 2011).

Por otro lado, las reuniones entre compañeros y familiares en torno a un asador, costumbre muy característica en esta capital del norte del país, parece que poco a poco se irá perdiendo, pues aunque la mayoría de los conflictos armados se han dado durante el día, la noche sigue generando ese característico temor de criminalidad y riesgo.

Adicionalmente a esto, durante el trascurso del día los ciudadanos se comparten información sobre la situación que se vive en diferentes puntos de la ciudad, y medios electrónicos como twitter o 
facebook se convierten en herramientas de supervivencia y resguardo ante posibles riesgos o puntos de conflicto. Aunque la población lucha por continuar sus actividades con la mayor tranquilidad, es necesario aclarar que la adopción de este tipo de actuaciones ya rompe con la cotidianidad de sus vidas, dejando de ser parte de una realidad externa y convirtiéndose en un elemento que les mueve, les motiva y les lleva a comportarse bajo nuevos parámetros de valor.

Para los ciudadanos que viven y desempeñan sus actividades en alguna de las ciudades que se encuentran en situación de riesgo, sus comportamientos pudieron haber marcado claros cambios al inicio del conflicto, sin embargo, después de haber pasado más de cinco ańos, la población parece acostumbrarse a aquello que no tenía cabida en sus posibilidades, pero que hoy, lamentable e inevitablemente, forma parte de su vida.

\section{LA ACCIÓN HUMANA ANTE LA PERSPECTIVA DE LA TEORÍA ELSTERIANA DE LA RACIONALIDAD}

Desde hace 40 años, en que se doctoró en Letras y Ciencias Sociales por la Universidad de París (1972), Jon Elster, filósofo y teórico social y político noruego, ha hecho grandes contribuciones a diferentes campos de las ciencias sociales, incluyendo el ámbito de la economía, la política y la filosofía. Sus aportaciones abarcan diferentes materias, pero sin duda, es su perspectiva sobre la racionalidad y la actuación humana, el punto de encuentro más significativo de la mayoría de sus contribuciones.

Para Elster, la acción se manifiesta como el resultado de un elaborado proceso reflexivo, en el que participan todos los elementos constitutivos de la misma, respetando las líneas causales generadoras de cada uno de estos elementos. La acción vista desde esta concepción debe implicar tres operaciones de optimización: hallar la mejor acción para las creencias y los deseos dados; formar la creencia lo mejor fundada para una prueba dada; $y$ acumular la cantidad atinada de pruebas para los deseos dados y las creencias previas (Elster 1999, 13). Es decir, que para la propuesta elsteriana, toda propuesta que busque explicar la actuación humana, deberá primero profun- 
dizar en los elementos de la acción, considerando el deseo que la mueve y las creencias que la respaldan.

La acción elsteriana considera que todo acto humano nace a partir de un deseo, el cual fijando los fines directos de la acción, permite que el individuo considere, a partir de sus creencias, las posibles acciones que se pueden realizar para alcanzar dichos fines. En este proceso cada una de las alternativas de actuación deberán irse acomodando para formar una escala de preferencias, la cual, logrará su posicionamiento a partir de las mismas creencias con las que cuenta el individuo. Una vez, que se tiene la escala plenamente estructurada se procederá a la elección de aquella opción que se considere sea la mejor según los fines señalados por el deseo, para que de tal forma, el agente llegue a una actuación, consciente y plena, sabiendo que lo que hace es lo que es más acorde a su historia de vida.

Un punto que se vuelve primordial en esta percepción elsteriana es el papel que desempeñan las creencias al fundamentar las opciones de actuación, ya que es importante considerar que no se puede dar una acción sin una elección previa y no se puede llegar a elegir en aquella situación en que no se tengan por lo menos dos opciones que creamos son óptimas y convenientes para nuestros fines. De tal manera, para llegar a una acción racional, el proceso natural sería el elegir entre dichas alternativas a aquella que nos resulta más óptima, considerando que tal evaluación se da conforme a nuestras creencias y a nuestra escala de preferencias.

Se debe hacer hincapié en que para que las alternativas de actuación, y por ende la elección, sean realmente racionales, es necesario que dichas creencias se encuentren debidamente fundamentadas, pues Jon Elster considera que solamente será a partir de la relación que existe entre las creencias y la evidencia que se posee de las mismas, que éstas pueden ser consideradas racionales y, por ende, cercanas a la realidad.

Por ello, la modificación de las creencias es un tema tan importante de ser explicado y comprendido, pues una nueva creencia puede desencadenar múltiples actuaciones que no esperaríamos cotidianamente de una persona, pero que se presume, tiene el respaldo suficiente para considerar que sigue actuando racionalmente. 


\section{LA RACIONALIDAD DE LOS NUEVOS COMPORTAMIENTOS}

Como se comentó con anterioridad, hace unos años en que la situación de algunas ciudades de México comenzó a complicarse, el comportamiento de su población era entendido como una respuesta a esta nueva amenaza que le afectaba. Sin embargo, conforme la intimidación fue formando parte de la realidad de los individuos, dicho comportamiento reactivo comenzó a entremezclarse con el resto de las actuaciones de los agentes, llegando al punto en que se diera un proceso de modificación del carácter de la población.

Semejante a lo que le sucede a un individuo de una población rural que se acostumbra después de unos meses al ajetreo de una gran urbe, los ciudadanos en situación de riesgo han tenido que acostumbrarse a su nueva realidad, modelando sus preferencias de actuación conforme algunos parámetros que consideran son beneficiosos para la realidad que enfrentan en este momento. A diferencia del comportamiento reactivo que se presentaba en un inicio, estas nuevas actuaciones se dan a partir de un proceso deliberativo, en el cual el agente, consciente de la situación que se vive en su entorno, decide actuar de cierta manera y no de otra que podría resultarle dañina.

De esta forma, la modificación del comportamiento que se ha dado en la población durante estos años puede dejar de responder únicamente a situaciones específicas, llevando a que las actuaciones cotidianas de las nuevas generaciones únicamente se den bajo los parámetros establecidos por la nueva realidad, misma que parece estar estructurando un sistema alternativo de creencias.

El manejar un perfil bajo, evitar llamar la atención, abstenerse de viajar solo o el alejarse de personas con ciertas características por considerar que pudieran ser peligrosas, son algunos de estos nuevos comportamientos, que si hace unos años podrían resultar excesivos o parte de un trastorno paranoide de la personalidad, hoy en día son la cotidianidad de las acciones de la población de ciertas ciudades.

Sin embargo, cabe el cuestionarse acerca de qué tan racional se está dando este proceso, es decir, ¿realmente se tiene la evidencia de que las nuevas actuaciones resultan ser las más óptimas?, ¿los nuevos comportamientos responden a un proceso racional de deliberación 
o acaso responden al miedo generalizado que se ha dado entre las masas durante los últimos años?

Para Jon Elster $(1988,30)$, la optimalidad de la evidencia que respalda las creencias es un problema toral que debe ser considerado al hablar de la racionalidad de las acciones, pues el grado de información que se requiere para sustentar una creencia no es fácilmente definible y tanto la falta como el exceso de evidencia, puede ser altamente perjudiciales en la generación de la escala de preferencias.

Por ello, el autor ha expresado que la caracterización positiva de las creencias racionales es la noción de juicio, ya que ésta permite que el agente sintetice la información vasta y difusa que puede llegar a tener sobre una idea, evitando que le dé una importancia o peso indebido a información irrelevante que pueden llegar a afectar la racionalidad de sus acciones.

Sin embargo, ¿cómo podemos llegar a hablar de un "buen juicio" cuando gran parte de las creencias que se tienen sobre la situación actual están basadas en el temor? Ésta es una duda que lleva a cuestionar las acciones mismas de la población, ya que en muchos de los casos parece ser que sus actuaciones, más que acercarlos a su objetivo de sentirse seguros, los ponen ante nuevas situaciones de riesgo. Parece ser que un juicio tan alterado, se convierte en un medio poco confiable para la generación de creencias, pues, éstas no logran estar, ni más cercanas a la verdad, ni más adecuadas a la realidad social, moral o individual del agente.

Este fallo en la relación entre evidencia-realidad está dando pauta a que la racionalidad de la actuación llegue a verse muy comprometida, afectando no únicamente a las creencias, sino también a las alternativas de actuación y, por ende, a la acción en general.

\section{LA EVIDENCIA DE LOS NUEVOS COMPORTAMIENTOS}

Mejor conocida como la sociedad de la información, la temporalidad actual parece bombardear al agente de diferente tipo de información que viene y va en todos los sentidos posibles. Opiniones a favor y en contra pueden ser encontradas dentro de un mismo medio y aunque parece que se tiene acceso a muchísima información, 
poca de ésta es realmente óptima y mucho menos, es indicada para convertirse en una evidencia que sustente las creencias de los individuos.

Cabe aclarar que Elster enfatiza que la evidencia no sólo debe estar respaldada en mucha información, sino que dicha información debe estar apegada a la realidad y no a un mundo teórico-ideal que aleja al individuo de su entorno. Por ello, la confiabilidad en los medios de información es determinante para los miembros de un Estado que se encuentra ante cambios tan considerables en su entorno.

Sin embargo, en un mundo contemporáneo en que la mayoría de la información que se recibe tiene una relación con los medios electrónicos, es difícil hacer una valoración precisa de la calidad de dicha información. Casos como lo sucedido en Tabasco en agosto del 2011, cuando un par de usuarios electrónicos causaron el temor en una población con información falsa (TabascoHoy 2011) o, bien, las múltiples infiltraciones de criminales informáticos en los distintos medios informativos, obliga a que la información generada por éstos deba ser analizada a consciencia, esta acción que pocas veces se hace.

Si a esto se le suma la filtración de noticias, la perspectiva de las diferentes agencias noticiosas e incluso las opiniones de los informantes, se puede ver que los medios de comunicación son una fuente de información muy cuestionable y que sin un segundo respaldo podría llegar a considerarse como evidencia poco óptima.

Por otro lado, otro medio de hacerse de evidencias sería la aportación de otros agentes o bien las vivencias del sujeto mismo, sin embargo, considerando que los hechos están comúnmente rodeados de situaciones de riesgo, violencia y peligrosidad, la naturaleza humana podría afectar rotundamente la objetividad de lo vivido, dando pauta a mucha información, pero igualmente muy subjetiva.

De esta manera, la falta de evidencia que respalde las nuevas creencias puede poner en entredicho el que las mismas estén racionalmente fundamentadas y que los nuevos comportamientos que respaldan sean realmente los más óptimos para satisfacer los deseos del agente. Para lograr una comprensión mayor de esto, se analizará 
el siguiente ejemplo; una joven mujer desde que comenzaron a aumentar los robos de automóviles teme por su seguridad, pues cree que ella podría ser presa de esta situación. Por ende y con el deseo de evitar ser robada, cambió su carro de lujo por un automóvil más austero y usado, esto con el fin de, según ella, llamar menos atención. ¿ ¿Será esta actuación racionalmente óptima?

Apoyando su decisión, se podría casi asegurar que el mantener un perfil bajo y llamar poco la atención podría reducir significativamente la posibilidad de ser robada, sin embargo, contrario a lo que ella piensa, según los últimos datos de la Asociación Mexicana de Instituciones de Seguros (AMIS), los automóviles mas robados durante el pasado 2011 no fueron los coches de lujo, sino los autos sencillos, por lo que en lugar de acercarse a la satisfacción de su deseo de evitar ser robada, esta joven mujer parece haber tomado la decisión contraria, poniéndose en una situación mucho mas riesgosa de la que tenía anteriormente (Escamilla 2011).

De igual forma se encuentran aquellos que evitan rotundamente salir de noche de sus hogares para evitar quedar en medio de una confrontación de delincuentes, sin embargo, si algo se ha podido apreciar en los últimos años, es que la mayoría de los enfrentamientos entre bandas del crimen organizado se han dado durante el día y aunque claro está que el evitar salir de noche puede disminuir la posibilidad de estar en dicha situación, esto no es tan óptimo como se cree.

Como estos ejemplos, hay muchas creencias que han dado pauta a diferentes comportamientos entre la población de las ciudades más golpeadas por la criminalidad y la violencia, sin embargo, como se ha podido apreciar, no todas las acciones son racionalmente óptimas, pues, en lugar de responder a una evidencia precisa, lo hacen a temores, miedos y subjetividades que afectan su juicio y alteran sus creencias.

La propuesta de racionalidad elsteriana, es un medio preciso que puede ayudar a que los individuos verifiquen que sus comportamientos realmente responden a los deseos que pretenden y no, como se pudo apreciar en el ejemplo de la joven mujer, lleven a un efecto contrario. 


\section{Comentarios finales}

En los últimos cinco años, parece ser que el México al que estábamos acostumbrados ya no es el mismo de antes. La violencia ha salido de las zonas donde se encontraba localizada $y$, ahora, se ha esparcido por la mayoría de las regiones, ciudades y calles del país. Esta situación ha llevado a que la población tenga que modificar la forma en que enfrenta a esta nueva realidad, para la cual no estaba preparada.

Las nuevas creencias que se tienen respecto a esta situación, llevan a que se generen nuevas opciones de actuación que permitan, según los ciudadanos, protegerse a ellos mismos, a los suyos y sus bienes. Sin embargo, al haber nacido como resultado del miedo, el temor y la urgencia, muchos de estos nuevos comportamientos tienen una fundamentación distorsionada que en lugar de acercar al agente a la satisfacción de sus deseos de tranquilidad, lo aleja cada vez más de la consecución de los mismos.

De manera adicional se le suma la enorme cantidad de información, que de manera poco objetiva, se presenta ante la población por medio de vías informales, que en lugar de respaldar las nuevas creencias con una evidencia óptima, la fundamentan con evidencia subjetiva, sesgada y deficiente, que en lugar de mejorar la comprensión del comportamiento del agente, lo vuelven mucho más confuso.

Jon Elster, filósofo y teórico social, propone que para llegar a tener actuaciones racionalmente óptimas, es necesario que el individuo se acerque a la comprensión de los elementos que constituyen su acción, es decir, que logre visualizar el deseo que trata de satisfacer, así como las acciones con las que busca hacerlo. Esta claridad de sus acciones, llevará a que el agente sea más consciente de sus actos, pudiendo valorar si los mismos responden a sus pretensiones y no únicamente a sus emociones, pasiones y miedos.

Es comprensible el hecho de que en un primer momento, la población reaccionara de una forma irracional y desordenada, pues, ante toda crisis, la urgencia lleva a la precipitación y al apremio, sin embargo, después de seis años en que la situación de violencia se ha encrudecido, es momento que se retome el camino de la racionali- 
dad, adoptando acciones concretas y realmente óptimas, desechando aquellas que no llevan a nada, sino únicamente a la confusión e irracionalidad de las acciones del agente.

\section{BibliografíA}

Ballinas, V., "Muertes de civiles en el combate al crimen, daños colaterales: Galván", La Jornada, 13 de abril de 2010, 5.

ВвС, “Los tres estados mas violentos de México en el 2010", 1 de enero de 2011, recuperado el 15 de marzo de 2012, de ввс Mundo, http://www.bbc.co.uk/mundo/ultimas_noticias/2011/01/ 110101_ultnot_mexico_estados_violentos_fp.shtml

CaOrsi, C., "La filosofía de la acción de Donald Davidson", en G. Leyva, Filosofía de la acción, Madrid, Universidad Autónoma Metropolitana, Editorial Síntesis, 2008, 653-684.

Davidson, D., Ensayos sobre acciones y sucesos, Barcelona, Crítica, 1995.

El Universal, "Atentados en Morelia, EU ve 'narcoterrorismo en cobardes ataques", 18 de septiembre de 2008, recuperado el 15 de marzo de 2012, El Universal.com.mx, http://www.eluniversal. com.mx/nacion/162452.html

ElSTER, J., Uvas amargas. Sobre la subversión de la racionalidad, Barcelona, Península, 1988. 1989.

Ulises y las sirenas, México, Fondo de Cultura Económica,

, "Estudios sobre la racionalidad", Lectura pronunciada en la ceremonia en que se le concedía el título de Doctor Honoris Causa por la Universidad de Valencia, Valencia, 1995.

Egonomics: análisis de la interacción entre racionalidad, emoción, preferencias y normas sociales en la economia de la acción individual y sus desviaciones, Barcelona, Gedisa, 1997. , Juicios salomónicos, Barcelona, Gedisa, 1999. , La explicación del comportamiento social: más tuercas y tornillos para las ciencias sociales, Barcelona, Gedisa, 2010.

Escamilla, V. M., "De los 10 autos más robados en México", $C N N$ expansión, 23 de agosto de 2011, recuperado el 16 de marzo de 
2012, http://www.cnnexpansion.com/mi-dinero/2011/08/23/ los-10-autos-mas-robados-en-mexico

Hernández, P., "Azota el flagelo del crimen a negocios y comercios de Monterrey", Acento Veintiuno, 6 de julio de 2011, recuperado el 15 de marzo de 2012, http://www.acentoveintiuno. com/?Azota-el-flagelo-del-crimen-a

Mendoza, E., "Cinco años de guerra, 60 mil muertos", Proceso. com.mx, 10 de diciembre de 2011, recuperado el 15 de marzo de 2012, http://www.proceso.com.mx/?p=290774

TabascoHoy, "Tuiteros de Veracruz enfrentan cargos por terrorismo", 1 de septiembre de 2011, recuperado el 15 de marzo de 2012, http://www.tabascohoy.com/noticia.php?id_nota=220249 